\title{
OTIMIZAÇÃO DAS CONDIÇÕES DE FERMENTAÇÃO EM ESTADO SÓLIDA E AVALIAÇÃO DAS ATIVIDADES ENZIMÁTICAS DE PAPEL FILTRO (FPU).
}

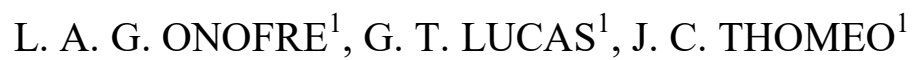 \\ ${ }^{1}$ Universidade Estadual "Julio de Mesquita Filho, Departamento de Engenharia e Tecnologia \\ de Alimentos \\ E-mail para contato: Leonardo_aredio@hotmail.com
}

\begin{abstract}
Resumo - Resumo - O presente trabalho buscou otimizar as condições de cultivo em estado sólido para produção de enzimas de celulolíticas variando a proporção dos substratos (bagaço de cana e farelo de trigo) e o pH da solução nutriente, buscando assim uma condição ótima de cultivo. Observou-se que quando o $\mathrm{pH}$ encontrasse no valor de 5 e a que a proporção de farelo de trigo é inferior a $20 \%$ a atividade de papel filtro (FPU) que é o conjunto de 3 celulases, foi o mais satisfatório pois chegou a índices de 110,896 U/gss.
\end{abstract}

\section{INTRODUÇÃO}

Devido a escassez de combustíveis fosseis o etanol tem sido considerado uma alternativa de matriz energética mais barata e mais "limpa". Sua produção pode ser oriunda de varias fontes de matéria prima vegetal. No Brasil a mais utilizada é a cana de açúcar com isso ocupando uma aposição de liderança na produção mundial de etanol (PACHECO, 2011).

O etanol convencional $(1 \mathrm{G})$ é obtido através da fermentação de açúcares extraído das matrizes vegetais, onde tem a sacarose e o amido como fonte de carbono como para iniciar a fermentação, processo esse similar a produção de bebidas alcoólicas. Já o etanol celulósico (2G) tem como fonte de carbono os açúcares provenientes da quebra das cadeias de celulose e hemicelulose, encontrados em biomassa descartada pelas agroindústrias, que no presente trabalho utilizou o bagaço de cana e o farelo de trigo.

No entanto para a produção do etanol $2 \mathrm{G}$ a partir dessa biomassa necessita do prétratamento do bagaço, onde é necessário a hidrólise das fibras do bagaço para que ocorra a liberação dos açúcares fermentescíveis, que pode ser feita de duas formas, por meio químico, que tem alta eficiência, porém resulta em uma grande quantidade de resíduos tóxicos que requerem maiores esforços na hora do descarte, ou a hidrólise enzimática, que é proveniente de enzimas celulolíticas e hemicelulolíticas obtidas a partir de fungos filamentosos (Kamm \& Kamm, 2004).

O processo de produção de enzimas por fungos filamentosos têm sua eficácia comprovada devido a suas necessidades para cultivo serem compatíveis com as condições oferecidas pelas técnicas de fermentação em estado sólido (FES), já que a mesma consiste em crescimento de micro-organismos sobre partículas porosas e úmidas, uma vez que o líquido 
contido na matriz sólida assegure o crescimento e metabolismo celular (Couto; Sanroman, 2006; Soccol; Vandenbergh, 2003; Palma, et al, 2000).

Neste contexto o presente trabalho tem como objetivo otimizar as condições de cultivo em estado sólido do fungo filamentoso Myceliophthora thermophila I-1D3b variando as proporções de substrato (bagaço de cana e farelo de trigo) e o pH da solução nutriente que foi adicionada ao meio de cultivo na busca por maiores atividades enzimática do conjunto de 3 celulases expressas pela FPU.

\section{MATERIAL E MÉTODOS}

Para o desenvolvimento experimental foi utilizado o Laboratório de Engenharia de Processos e Biorreatores do Instituto de Biociências, Letras e Ciências Exatas de São José dom Rio Preto - IBILCE / UNESP.

\subsection{Microrganismo}

O microrganismo utilizado foi o fungo termofílico Myceliophthora thermophila I-1D3b, isolado pela Prof $^{\mathrm{a}} \mathrm{Dr}^{\mathrm{a}}$ Daniela Alonso Bocchini, pertencente do Instituto de Química da Unesp, encontrado em pilhas de bagaço de cana-de-açúcar. A cepa do fungo I-1D3b pertence à coleção do Laboratório de Microbiologia e Bioquímica Aplicada do IBILCE-Unesp, encontrando-se hoje câmara de congelamento à $-80^{\circ} \mathrm{C}$. Para a realização do inóculo foi utilizado a cultura em suspensão de esporos, obtida após a replicação das cepas em erlenmeyers com meio de cultura de Ágar Sabouraud Dextrose (ASD) mantidas em câmara de $\mathrm{BOD}$ a $45^{\circ} \mathrm{C}$ durante 72 horas. Após esse período foi adicionado solução nutriente conforme utilizada por Zanelato (2011) até obter-se uma concentração de $10^{7}$ esporos $/ \mathrm{mL}$.

\subsection{Substratos e Atividade Enzimática}

Os substratos empregados foram o bagaço de cana-de-açúcar e farelo de trigo. O bagaço doado pela Usina Virgolino de Oliveira situada na cidade de José Bonifácio-SP, seco em estufa de circulação forçada a $60^{\circ} \mathrm{C}$ até atingir peso constante, para a padronização do tamanho das fibras o bagaço foi peneirado em peneiras de 4 e $3 \mathrm{~mm}$. O farelo de trigo obtido no comércio local, seco na estufa de circulação forçada a $40^{\circ} \mathrm{C}$ até peso constante.

Para realização dos ensaios os substratos foram acondicionado em embalagem plástica de polipropileno em diferentes proporções Bagaço de Cana e Farelo de Trigo (BC:FT) como mostra a Tabela 1, totalizando $5 \mathrm{~g}$, na abertura das embalagens foi acoplado fragmentos de cano de PVC com $4 \mathrm{~cm}$ de diâmetro e $5 \mathrm{~cm}$ de comprimento sendo tampados com tampões de algodão e gaze e esterilizado em autoclave a $121^{\circ} \mathrm{C}, 1,1 \mathrm{ATM}$ por $20 \mathrm{~min}$. 
Tabela 1 - Planejamento Experimental (DCCR) variando a proporção de bagaço de cana $(\mathrm{BC})$ e farelo de trigo (FT) e o pH da solução nutriente.

\begin{tabular}{|c|c|c|}
\hline Ensaio & ph & $\begin{array}{c}\text { Formulação } \\
\text { (BC:FT) }\end{array}$ \\
\hline \hline 1 & 4 & $06: 04$ \\
\hline 2 & 6 & $6: 4$ \\
\hline 3 & 4 & $8: 2$ \\
\hline 4 & 6 & $8: 2$ \\
\hline 5 & 6,4 & $7: 3$ \\
\hline 6 & 3,6 & $7: 3$ \\
\hline 7 & 5 & $8,4: 1,6$ \\
\hline 8 & 5 & $5,6: 4,4$ \\
\hline 9 & 5 & $7: 3$ \\
\hline 10 & 5 & $7: 3$ \\
\hline 11 & 5 & $7: 3$ \\
\hline
\end{tabular}

Após a esterilização, a umidade das amostras foram elevada a $70 \%$ com a adição de solução nutriente, respeitando em cada ensaio o $\mathrm{pH}$ determinando no planejamento, mais inóculo e acondicionada em BOD a $\pm 45^{\circ} \mathrm{C}$ por 96 horas. Foram retiradas amostras nos tempos de 0 horas, 24 horas, 48 horas e 96 horas para a extração do extrato enzimático e posterior análise de atividade enzimática. Para a realização da extração foi adicionado $20 \mathrm{~mL}$ de água destilada para cada grama de substrato sólido seco, agitado por 30 minutos, filtrado e centrifugado a $10.000 \mathrm{rpm}$ por $15 \mathrm{~min}$. Com o sobrenadante foi feita a avaliação da atividade enzimática de endoglucanase. A determinação foi feita segundo Pinto (2010), com modificações, esta reação resultará na liberação de açúcares redutores utilizou-se a metodologia de quantificação segundo Miller (1959) onde para definir a unidade de atividade de enzimática a quantidade necessária para liberal $1,0 \mu \mathrm{mol}$ de glicose por minuto de reação por $\mathrm{mL}$ de enzima de acordo coma curva padrão de glicose, resultando em unidades por grama de substrato seco (U/gss). Em se tratando de fermentação em estado sólido, com base no volume de água utilizado para extração das enzimas, os resultados das atividades enzimáticas foram convertidos para serem expressos em unidades por grama de substrato sólido (U/gss).

\section{RESULTADOS E DISCUSSÃO}

Após o período de 96 horas de fermentação, foram feitas as extrações e avaliada as atividades enzimáticas de endoglucanase. A Figura 1 nos mostra o comportamento durante as 96 horas de fermentação, podemos observar que no ensaio onde o $\mathrm{pH}=5$ os resultados obtidos foram mais satisfatórios, o que é explicado pela literatura. Porém vale ressaltar que a proporção de BC:FT que obteve melhor resultado foi a que continha uma porcentagem maior de bagaço de cana do que a de farelo de trigo. 
Figura 1 - Atividade enzimática de papel filtro (U/gss) ao longo de 96 horas de fermentação nos 11 ensaios.

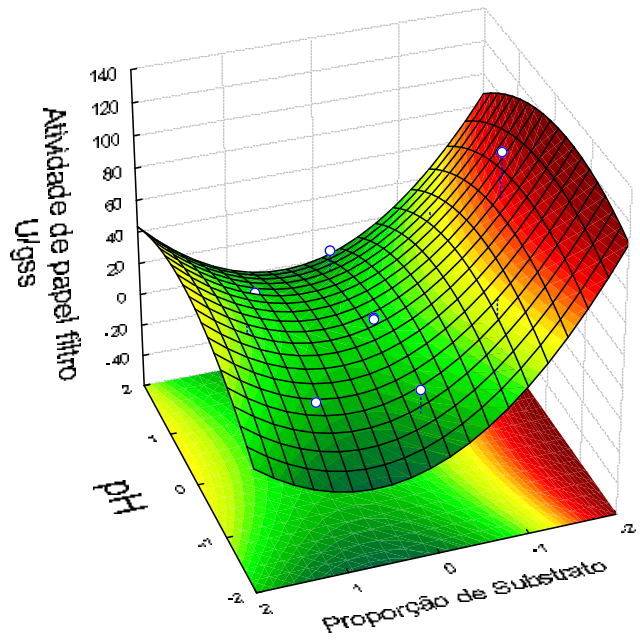

\section{CONCLUSÃO}

De ante os dados obtidos pode-e observar que quando o $\mathrm{pH}$ da solução nutriente estava no valor de 5 e a proporção de farelo de trigo inferior a $20 \%$ do total da formulação proposta os resultados de atividade de papel filtro, que nada mais é do que a ação conjunta de três celulases, chegou em valores de junção de três enzimas mais acido a atividade enzimática foi maior, acredita-se que esse resultado explica-se pois em $\mathrm{pH}$ mais básicos difere das condições ótimas de crescimento do fungo dificultando assim o seu crescimento e a secreção da enzima.

\section{REFERÊNCIAS}

COUTO, S. R.; SANROMÁN, M. A. Application of solid-state fermentation to food industry - A Review. Journal of Food Engineering, Califórnia, v. 76, n. 3, p. 291-302, 2006.

KAMM, B.; KAMM, M. Principlesofbiorefineries. Applied Microbiology and Biotechnology, v.64, n.2, p.137-145, 2004. ISSN 0175-7598.

MILLER, G.L. Use of dinitrosalicylic acid reagent for determination of reducing sugar. Analitical Chemistry, v. 31, p.426 - 428, 1959.

PACHECO, T. F. Produção de etanol: primeira ou segunda geração?. Circular Técnica 04, Brasília: Embrapa, 2011.

PALMA, M.B., PINTO, A.L., GOMBERT, A.K., SEITZ, K.H., KIVATINITZ, S.C., CASTILHO, L.R., FREIRE, D.M.G. 2000. Lipase production by Penicillium estrictumusing solid waste of industrial babassuoilproduction as substrate. Appl Biochem Biotech, 84, 1137- 1145. 
PELIZER, L. H.; PONTIERI, M. H.; MORAES, I. O. Utilização de resíduos agroindustriais em processos biotecnológicos como perspectiva de redução do impacto ambiental. Journal of Technology Management \& Innovation, Santiago, v. 2, n. 1, p. 118-127, 2007.

PINTO, T. O. P. Produção de enzimas celulolíticas pelos fungos Thermoascus aurantiacus CBMAI 756, Thermomyces lanuginosus, Trichoderma reesei QM9414 e Penicillium viridicatum RFC3 e aplicação na sacarificação do bagaço de cana-de-açúcar com diferentes pré-tratamentos. 2010. 91f. Dissertação (Mestrado) - Instituto de Biociências, Letras e Ciências Exatas, Universidade Estadual Paulista “Júlio de Mesquita Filho", São José do Rio Preto, 2010.

SOCCOL, C. R.; VANDENBERGHE, L. P. S. Overview ofappliedsolid-statefermentation in Brazil. Biochemical Engineering Journal, Amsterdan, v. 13, n. 2/3, p. 205-218, 2003.

ZANELATO, A. I. Produção de enzimas celulolíticas por fermentação em estado sólido em bioreator de leito fixo. Dissertação (Mestrado). Universidade Estadual Paulista, São José do Rio Preto, 2011. 